\title{
The association between time scarcity, sociodemographic correlates and consumption of ultra-processed foods among parents in Norway: a cross-sectional study
}

Ingrid Laukeland Djupegot ${ }^{\dagger}$, Camilla Bengtson Nenseth ${ }^{\dagger}$, Elling Bere, Helga Birgit Torgeirsdotter Bjørnarå, Sissel Heidi Helland, Nina Cecilie Øverby, Monica Klungland Torstveit and Tonje Holte Stea*

\begin{abstract}
Background: Use of ultra-processed foods has expanded rapidly over the last decades and high consumption has been positively associated with risk of e.g. overweight, obesity and type 2 diabetes. Ultra-processed foods offer convenience as they require minimal time for preparation. It is therefore reasonable to assume that such foods are consumed more often among people who experience time scarcity. The main aim of this study was to investigate the association between time scarcity and consumption of ultra-processed foods among parents of 2-year olds in Norway. A secondary aim was to investigate the association between sociodemographic correlates, weight status and consumption of ultra-processed foods.
\end{abstract}

Methods: This cross-sectional study included 497 participants. Chi-square and cross tabulations were used to calculate proportions of high vs. low consumption of ultra-processed foods in relation to time scarcity, sociodemographic correlates and weight status. Binary logistic regression analyses were performed to test the relationship between independent variables and consumption of ultra-processed foods.

Results: Participants reporting medium and high time scarcity were more likely to have a high consumption of ultra-processed dinner products $(\mathrm{OR}=3.68,95 \% \mathrm{Cl}=2.32-5.84$ and $\mathrm{OR}=3.10,1.80-5.35$, respectively) and fast foods $(O R=2.60,1.62-4.18$ and $O R=1.90,1.08-3.32$, respectively) compared to those with low time scarcity. Further, participants with medium time scarcity were more likely to have a high consumption of snacks and soft drinks compared to participants with low time scarcity $(\mathrm{OR}=1.63,1.06-2.49)$. Finally, gender, ethnicity, educational level, number of children in the household and weight status were identified as important factors associated with the consumption of certain types of ultra-processed foods.

Conclusions: Results from the present study showed that time scarcity, various sociodemographic factors and weight status was associated with consumption of processed foods. Future studies with a longitudinal design are needed to further explore these patterns over a longer period of time.

Keywords: Ultra-processed foods, Processed foods, Time scarcity, Convenience, Adults, Parents

\footnotetext{
*Correspondence: tonje.h.stea@uia.no

${ }^{\dagger}$ Equal contributors

Department of Public Health, Sport and Nutrition, University of Agder,

Postboks 422, 4604 Kristiansand, Norway
} 


\section{Background}

Highly processed foods have been classified as ultra-processed by Monteiro et al. in the NOVA ${ }^{1}$ food classification system, and include products that are industrially manufactured and usually highly accessible, attractive, palatable and habit-forming [1]. The NOVA classification categorizes foodstuffs in four groups based on the extent and purpose of processing; Unprocessed or minimally processed foods, processed culinary ingredients, processed foods, and ultraprocessed foods (UPF) [1, 2]. Group 4 products (ultraprocessed foods) are composed of industrial ingredients (e.g. corn syrup, lactose, soy proteins) and culinary ingredients that are refined or extracted from whole foods (e.g. flour, oil, sugar) $[1,2]$. UPF are often referred to as convenience foods or fast foods, and examples include readymeals, soft drinks, chocolate and chips [1]. Furthermore, UPF are typically energy-dense, low in dietary fibre, protein and micronutrients, and they often contain more sugar, sodium and fat/saturated fat than unprocessed and minimally processed foods [3-5]. An excess intake of UPF might therefore have severe implications for human health, and has been linked to several lifestyle related diseases including obesity, type 2 diabetes, metabolic syndrome, cardiovascular disease and cancer in children, adolescents and adults [6-16]. During the twentieth century, whole and fresh foods have increasingly been replaced with convenient pre-prepared and ready-to-eat products that require minimal preparation $[3,17]$. A large prospective study conducted in ten European countries found that highly processed foods contributed with $61-79 \%$ of mean energy intake [18]. These results are consistent with findings from Canada and the United States, where approximately 60\% of household food expenditure and mean energy intake was explained by purchasing and consumption of highly processed foods and beverages [19-21].

A range of factors might influence the use of UPFproducts, and among these are time scarcity, which has previously been described as people's perceptions or feelings of not having enough time to do all they want or need to in a day (Godbey, Lifset \& Robinson, 1998, in Jabs \& Devine 2006 p. 197) [22]. Families with children often operate on a tight schedule juggling work, domestic work and leisure activities [22, 23]. Qualitative studies have reported that employed mothers often experience a general lack of time, which also influence their food choices $[23,24]$. Preparation of healthy foods was perceived to be a time-consuming activity, and thereby highly processed convenience foods were often used as a time saving strategy [23, 24]. Although qualitative research has indicated that feelings of time scarcity might contribute to less home-prepared meals with fresh ingredients and an increased use of e.g. ready-meals and fast foods, there is a lack of consistent quantitative evidence regarding this association [25]. As parents' behaviour might influence the eating habits of their children [26, 27], investigating the influence of time scarcity on the use of convenience foods and fast foods among parents is of particular interest. Furthermore, quantitative studies have reported sociodemographic differences in ultra-processed food consumption (UPFC) [28, 29]. For illustration, low socioeconomic status has been associated with less healthy diets, including higher consumption of fast foods and soft drinks [30-33]. On this basis, it is appropriate to adjust for sociodemographic variables when investigating factors potentially influencing UPFC.

The main aim of this study was to investigate the association between time scarcity and UPFC among parents of 2-year olds in Norway. A secondary aim was to investigate the association between sociodemographic correlates (gender, ethnicity, education and number of children in the household), weight status and UPFC.

\section{Methods \\ Design and study sample}

This cross-sectional study is part of The Healthy and Sustainable Lifestyle project and the Child Food Courage project. Registration code for the Child Food Courage Project is 37,459. Data were collected between October 2014 and January 2015. The study was conducted in accordance with the Helsinki Declaration, and permission to collect and store data was obtained from Norwegian Social Science Data Services. Furthermore, written consent was electronically provided by the participants prior to data collection. About 3100 parents in the counties of Aust-Agder and Vest-Agder in Southern Norway, with children born in 2012, received information about the project through their kindergarten. Participants completed a web-based questionnaire (Additional file 1), which comprised a food frequency questionnaire and included questions about lifestyle behaviours, self-perceived health and quality of life among parents of toddler's. In total, 605 parents signed up to participate. Only participants who completed the questionnaire $(n=497)$ were included in the current study, which yielded a response rate on approximately $16 \%$.

\section{Outcome measures}

Questions from the Healthy and Sustainable Lifestylesurvey were used to develop three scores to measure UPFC; Ultra-processed dinner products, sweet/salty snacks \& soft drinks and fast foods away from home. The selection of questions was based on the NOVA classification of food products proposed by Monteiro et al. $[1,34]$. There are currently no dietary recommendations regarding UPF in Norway that can inform the operationalisation of UPFC. For all outcome scores, cut-offs were therefore estimated to get the most equally sized groups to increase the statistical power, and variables were dichotomized into low vs. high consumption. 


\section{Ultra-processed dinner products}

This score comprised 5 items measuring frequency of consumption of ready-to-eat/pre-prepared dinner products. Questions included How often do you eat ... Noodles; Ready meals; Sausages; Pommes frites; Dinners based on minced meat (e.g. tacos, pasta). Response alternatives were assigned different values, and ranged from never to every day (never $=0$, less than once a month $=0.25,1-3$ times/ month $=0.5$, once a week $=1,2$ times $/$ week $=2,3$ times $/$ week $=3,4$ times $/$ week $=4,5$ times $/$ week $=5,6$ times $/$ week $=6$, every day $=7$ ). Total score ranged from 0 to 35 , with higher score indicating a higher consumption of ultra-processed dinner products.

\section{Sweet/salty snacks \& soft drinks}

This score comprised 4 items measuring frequency of consumption of sweet/salty snacks and soft drinks. Questions included How often do you eat ... Salted snacks (e.g. chips, cheese doodles, salted nuts); Confectionery (e.g. sweets, chocolate), and How often do you drink ... Sugar sweetened beverages (e.g. soft drinks, juice, ice tea, ice coffee); Artificially sweetened beverages (e.g. diet soft drinks, diet juice, diet ice tea). Response alternatives were assigned different values, and ranged from never to several times a day (never $=0$, less than once a week $=0.5$, once a week $=1,2$ times $/$ week $=2,3$ times $/$ week $=3,4$ times $/$ week $=4$, 5 times $/$ week $=5,6$ times $/$ week $=6$, every day $=7$, several times a day $=10$ ). Total score ranged from 0 to 40 , with higher score indicating a higher consumption of snacks \& soft drinks.

\section{Fast foods away from home}

This score comprised 2 items measuring frequency of consumption of food from fast food restaurants, gas stations and convenience stores. Questions included How often do you eat food from fast food restaurants (e.g. McDonalds, takeaway-restaurants) and How often do you eat food bought at a gas station/convenience store (e.g. 7-eleven). Response alternatives were assigned different values, and ranged from never to every day (never $=0$, less than once a week $=0.5$, once a week $=1,2$ times $/$ week $=2,3$ times $/$ week $=3$, 4 times $/$ week $=4,5$ times $/$ week $=5,6$ times $/$ week $=6$, every day $=7$ ). Total score ranged from 0 to 14 , with higher score indicating a more frequent consumption of fast foods.

\section{Independent variables}

\section{Time scarcity}

Van der Lippe's adjusted version of Garhammer's index of time pressure, was used to measure time scarcity $[35,36]$. In this 7 item scale, study participants were asked to what extent the following statements coincided with their experiences: I am under time pressure, I wish I had more time for myself, I feel I am under time pressure from others, I cannot deal with important things properly due to lack of time, I cannot get proper sleep, I cannot recover properly from illness due to lack of time and I am under so much time pressure that my health suffers. Response alternatives ranged from never to always $(1=$ never, 2 = rarely, $3=$ sometimes, $4=$ often, $5=$ always). Total score ranged from 7 to 35, with higher score indicating a higher degree of time scarcity. In previous studies, the time pressure scale has shown a high level of internal consistency, with a Cronbach's alpha of 0.79 [35] and 0.87 [37]. Cut-offs were estimated to get the most equally sized groups, and the time scarcity variable was further trichotomized into a low, medium and high group.

\section{Sociodemographic correlates and weight status}

Gender (men vs. women), ethnicity (born in Norway vs. not born in Norway), educational level (higher education at university/college vs. no higher education), number of children in the household (2 vs. 1 and $\geq 3$ vs. 1 ) and weight status (BMI $\geq 25.0 \mathrm{~kg} / \mathrm{m}^{2}$ vs. $\leq 24.9 \mathrm{~kg} / \mathrm{m}^{2}$ ) were also tested as possible predictors of UPFC. Participants with BMI $\geq 25.0 \mathrm{~kg} / \mathrm{m}^{2}$ are referred to as overweight/obese, while participants with BMI $\leq 24.9 \mathrm{~kg} / \mathrm{m}^{2}$ are referred to as normal weight as there were few underweight participants $\left(\right.$ BMI $\left.<18.5 \mathrm{~kg} / \mathrm{m}^{2}\right)$.

\section{Statistical analyses}

All analyses were performed with the statistical software package IBM SPSS Statistics version 22.0 (IBM Corp., Somers, NY, USA.). Proportions of study participants having a high consumption of ultra-processed dinner products, sweet/salty snacks \& soft drinks and fast foods away from home, in relation to the proposed correlates, were calculated using cross tabulations and chi-square. Proportions classified with high time scarcity in relation to gender, ethnicity, educational level, number of children in the household and weight status were also calculated with cross tabulation and chi-square.

Binary logistic regression analyses were performed with appropriate sampling weights (for level of education and gender) to test the relationship between the independent variables (time scarcity, gender, ethnicity, educational level, number of children and weight status) and the dependent variables (consumption of ultra-processed dinner products, sweet/salty snacks \& soft drinks and fast foods away from home). The variables were entered into the model in two blocks: First, the unadjusted relationship of time scarcity and UPFC was tested, and then the sociodemographic correlates and weight status were included in the model. Results were regarded significant at $p<0.05$. 


\section{Results}

\section{Characteristics of study sample}

In our final sample, $90 \%$ of study participants were women and $90 \%$ were born in Norway. Age ranged from 20 to 46 years (mean $=32.2$ years), and mean Body Mass Index (BMI) was $24.9 \mathrm{~kg} / \mathrm{m}^{2}$. Regarding educational status, $69 \%$ had higher education at university/college level ( $<4$ years or $\geq 4$ years). A total of $33 \%$ of the study participants had one child living in the household, $47 \%$ had two children, and $20 \%$ had three or more children.

\section{Time scarcity}

In the present study, the time scarcity scale had a Cronbach's alpha of 0.87 . Mean score of experienced time scarcity in the study sample was $20.3 \pm 5.1$ (not reported in table). According to the results presented in Table 1, there were no differences between groups in the number of participants being categorized as having high degree of time scarcity. (men vs. women, ethnic Norwegian vs. non-ethnic Norwegian, overweight/obese vs. normal weight, higher education vs. no higher education, $2 / \geq 3$ children vs. 1 child). In the descriptive analyses, $35.7 \%$ of participants with low time scarcity, $49.2 \%$ of participants with medium time scarcity and $44.9 \%$ of participants with high time scarcity were categorized as high consumers of fast foods ( $p=0.037$, Table 1$)$. Weighted regression analyses adjusted for sociodemographic correlates and weight status showed that participants with both medium and high time scarcity were more likely to be high consumers of ultra-processed dinner products $(\mathrm{OR}=3.68,95 \% \mathrm{CI}=2$. 32-5.84 and $\mathrm{OR}=3.10,1.80-5.35$, respectively) and fast foods $(\mathrm{OR}=2.60,1.62-4.18$ and $\mathrm{OR}=1.90,1.08-3.32$, respectively) compared to those with low time scarcity (Table 2). Further, participants with medium time scarcity, but not high time scarcity, were more likely to be high consumers of snacks \& soft drinks compared to participants with low time scarcity $(\mathrm{OR}=1.63$, 1.062.49, Table 2).

\section{Sociodemographic correlates and weight status}

Table 1 showed no difference in the number of male and female participants being categorized as having high consumption of ultra-processed dinner products, snacks \& soft drinks and fast foods. Adjusted regression analyses, however revealed that men were more likely to be categorized as high consumers of ultra-processed dinner products and fast foods than women $(\mathrm{OR}=2.58,1.65-$ 4.06 and $\mathrm{OR}=3.78,2.35-6.08$, respectively).

A higher number of ethnic compared to non-ethnic Norwegians were categorized as having high consumption of snacks \& soft drinks (50.9\% vs $25.9 \%, p=0.001)$ and fast foods $(45.2 \%$ vs $29.6, p=0.029)$ (Table 1$)$, and the adjusted analyses confirmed that ethnic Norwegians were more likely to be categorized as high consumers of both ultra-processed dinner products $(\mathrm{OR}=1.98,1.11-$ 3.53), snacks \& soft drinks $(\mathrm{OR}=1.87,1.08-3.23)$ as well as fast foods $(\mathrm{OR}=4.79,2.50-9.15)$ compared to non-ethnic Norwegians.

Further, the results revealed that less participants with high education was categorized as having a high consumption of snacks \& soft drinks $(44.5 \%$ vs. $56.9 \%$, $p=0.011)$ and fast foods $(40.4 \%$ vs. $51.0 \%, p=0.028)$ compared to those with low education (Table 1). According to adjusted regression analyses, high educated participants were less likely to be categorized as high consumers of both ultra-processed dinner products $(\mathrm{OR}=0.61,0.40-0.92)$ and snacks \& soft drinks $(\mathrm{OR}=0.55,0.37-0.81)$ compared to those with low educational level (Table 2).

A higher number of overweight/obese participants was categorized as high consumers of snacks \& soft drinks compared to normal weight participants (54.3\% vs. $45.1 \%$, $p=0.044$, Table 1). Adjusted regression analyses did, however, not confirm an association between weight status and consumption of snacks \& soft drinks. On the other hand, the adjusted analysed showed that overweight/obese participants were more likely to be categorized as high consumers of ultra-processed dinner products $(\mathrm{OR}=1.54$, $1.04-2.30)$ and fast foods $(\mathrm{OR}=3.40,2.26-5.11)$ than normal weight participants.

Furthermore, descriptive analyses did not show a significant difference between the number of participants with one, two or three children in the household being categorized as having a high consumption of ultraprocessed dinner products, snacks \& soft drinks and fast foods. Results from adjusted analyses showed, however, a higher consumption of ultra-processed food products among participants with 3 or more children in the household compared to those with one child in the household ( $\mathrm{OR}=4.22,2.26-7.89)$.

\section{Discussion}

Time scarcity

Findings in the present study showed that time scarcity was associated with both consumption of ultraprocessed dinner products, snacks \& soft drinks as well as fast foods away from home after adjustment for sociodemographic correlates and weight status. Previously published studies have also suggested that time shortage is a barrier to healthy eating as those experiencing time pressure were more likely to eat fast food more frequently and less likely to meet the fruit and vegetable recommendations [38, 39]. In addition, time pressure, having a paid job and number of working hours have also been positively associated with the use of ready-meals such as frozen pizzas and TV dinners [40, 41], suggesting that use of ready- 
Table 1 Descriptive statistics of the association between time scarcity, gender, age, ethnicity, weight status, education, number of children and dichotomized indicators of ultra-processed food consumption Percentage of groups in high category of time scarcity, ultra-processed dinner products, sweet/salty snacks \& soft drinks and fast foods away from home. ${ }^{*} p<0.05$

\begin{tabular}{|c|c|c|c|c|c|}
\hline & & Time scarcity & Ultra-processed dinner products & Snacks \& Soft drinks & Fast foods away from home \\
\hline & $n$ & $\%$ in high category & $\%$ in high category & $\%$ in high category & $\%$ in high category \\
\hline All & 497 & 29.6 & 48.9 & 48.3 & 43.7 \\
\hline \multicolumn{6}{|l|}{ Time scarcity } \\
\hline Low & 157 & & 43.3 & 43.9 & 35.7 \\
\hline Medium & 193 & & 49.7 & 49.2 & 49.2 \\
\hline High & 147 & & 53.7 & 51.7 & 44.9 \\
\hline$p$ & & & 0.183 & 0.380 & $0.037^{*}$ \\
\hline \multicolumn{6}{|l|}{ Gender } \\
\hline Men & 52 & 21.2 & 57.7 & 44.2 & 55.8 \\
\hline Women & 445 & 30.6 & 47.9 & 48.8 & 42.2 \\
\hline$p$ & & 0.160 & 0.180 & 0.536 & 0.063 \\
\hline \multicolumn{6}{|l|}{ Ethnicity } \\
\hline Native & 442 & 30.3 & 50.5 & 50.9 & 45.2 \\
\hline Non-native & 54 & 24.1 & 37.0 & 25.9 & 29.6 \\
\hline$p$ & & 0.343 & 0.063 & $0.001^{*}$ & $0.029^{*}$ \\
\hline \multicolumn{6}{|l|}{ Weight status } \\
\hline Normal weight & 293 & 29.0 & 46.8 & 45.1 & 40.6 \\
\hline Overweight/obese & 197 & 29.9 & 52.8 & 54.3 & 49.2 \\
\hline$p$ & & 0.823 & 0.190 & $0.044^{*}$ & 0.059 \\
\hline \multicolumn{6}{|l|}{ Education } \\
\hline No higher education & 153 & 28.1 & 53.6 & 56.9 & 51.0 \\
\hline Higher education & 344 & 30.2 & 46.8 & 44.5 & 40.4 \\
\hline$p$ & & 0.631 & 0.162 & $0.011^{*}$ & $0.028^{*}$ \\
\hline \multicolumn{6}{|c|}{ Number of children in the household } \\
\hline 1 & 163 & 28.2 & 47.2 & 48.5 & 44.8 \\
\hline 2 & 231 & 30.7 & 48.1 & 47.2 & 42.9 \\
\hline$\geq 3$ & 101 & 28.7 & 53.5 & 51.5 & 44.6 \\
\hline$p$ & & 0.849 & 0.580 & 0.771 & 0.918 \\
\hline
\end{tabular}

meals might be a convenient way of managing time pressure. The positive association between time scarcity and use of ultra-processed dinner products shown in the present study supports the hypothesis that the consumption of ready meals is used as a strategy for better time management.

\section{Sociodemographic correlates and weight status}

In the current study, adjusted analyses showed that men were more likely to be classified as high consumers of ultra-processed dinner products and fast foods, but not snacks \& soft drinks, when compared to women. These results have to some extent been supported by previous studies showing an increased consumption of fast foods, sugar sweetened soft drinks and processed meat and a decreased consumption of sweets among men compared to women, [29, 33, 42].

Furthermore, adjusted analyses in the present study showed that participants with high educational level had $39 \%$ lower odds of consuming ultra-processed dinner products and $45 \%$ lower odds of consuming snacks \& soft drinks compared to those with low educational level. Whereas our study did not find a significant association between educational level and consumption of fast foods, Thornton et al. [31] found that low educational level, decreased household income and being a blue-collar employee was associated with an increase in fast food purchases. Similarly, Larson et al. [43] found that frequent fast food intake was most common among individuals with low- 
Table 2 Odds ratios for the associations between time scarcity, sociodemographic correlates, weight status and high consumption of ultra-processed foods

\begin{tabular}{|c|c|c|c|c|c|c|c|c|c|c|c|c|}
\hline & \multicolumn{4}{|c|}{ Ultra-processed dinner products } & \multicolumn{4}{|c|}{ Snacks \& Soft drinks } & \multicolumn{4}{|c|}{ Fast food away from home } \\
\hline & \multicolumn{2}{|c|}{ Model 1} & \multicolumn{2}{|c|}{ Model 2} & \multicolumn{2}{|c|}{ Model 3} & \multicolumn{2}{|c|}{ Model 4} & \multicolumn{2}{|c|}{ Model 5} & \multicolumn{2}{|c|}{ Model 6} \\
\hline & OR & $95 \% \mathrm{Cl}$ & $\mathrm{OR}$ & $95 \% \mathrm{Cl}$ & $\mathrm{OR}$ & $95 \% \mathrm{Cl}$ & $\mathrm{OR}$ & $95 \% \mathrm{Cl}$ & OR & $95 \% \mathrm{Cl}$ & $\mathrm{OR}$ & $95 \% \mathrm{Cl}$ \\
\hline \multicolumn{13}{|l|}{ Time scarcity } \\
\hline Medium (vs. low) & $3.04^{*}$ & $2.01-4.59$ & $3.68^{*}$ & $2.32-5.84$ & 1.46 & $0.98-2.17$ & $1.63^{*}$ & $1.06-2.49$ & $2.19^{*}$ & $1.46-3.28$ & $2.60^{*}$ & $1.62-4.18$ \\
\hline High (vs. low) & $2.66^{*}$ & $1.66-4.27$ & $3.10^{*}$ & $1.80-5.35$ & 1.36 & $0.86-2.16$ & 1.57 & $0.94-2.61$ & $1.62^{*}$ & $1.02-2.56$ & $1.90^{*}$ & $1.08-3.32$ \\
\hline \multicolumn{13}{|l|}{ Sex } \\
\hline Men (vs. women) & & & $2.58^{*}$ & $1.65-4.06$ & & & 1.08 & $0.71-1.62$ & & & $3.78^{*}$ & $2.35-6.08$ \\
\hline \multicolumn{13}{|l|}{ Ethnicity } \\
\hline Native (vs. non-native) & & & $1.98^{*}$ & $1.11-3.53$ & & & $1.87^{*}$ & $1.08-3.23$ & & & $4.79^{*}$ & $2.50-9.15$ \\
\hline \multicolumn{13}{|l|}{ BMl } \\
\hline Overweight/obese (vs. normal weight) & & & $1.54^{*}$ & $1.04-2.30$ & & & 1.18 & $0.81-1.71$ & & & $3.40^{*}$ & $2.26-5.11$ \\
\hline \multicolumn{13}{|l|}{ Education } \\
\hline $\begin{array}{l}\text { Higher education } \\
\text { (vs. no higher education) }\end{array}$ & & & $0.61^{*}$ & $0.40-0.92$ & & & $0.55^{*}$ & $0.37-0.81$ & & & 0.68 & $0.45-1.04$ \\
\hline \multicolumn{13}{|l|}{ Number of children in the household } \\
\hline 2 (vs. 1) & & & 1.33 & $0.86-2.07$ & & & 0.75 & $0.50-1.14$ & & & 0.86 & $0.54-1.38$ \\
\hline$\geq 3$ (vs. 1) & & & $4.22^{*}$ & $2.26-7.89$ & & & 1.75 & $1.00-3.08$ & & & 1.53 & $0.82-2.85$ \\
\hline \multicolumn{13}{|l|}{ Age } \\
\hline Continous (years) & & & $0.93^{*}$ & $0.89-0.97$ & & & 0.98 & $0.94-1.02$ & & & $0.90^{*}$ & $0.86-0.94$ \\
\hline
\end{tabular}

OR: Odds ratio; Cl: Confidence interval; ${ }^{*} p<0.05$

middle socioeconomic status, and another study has reported an association between dropping out of school before the age of sixteen and low levels of food involvement in the kitchen, resulting in a more frequent consumption of junk foods [44].

Results in the present study also showed higher odds of consuming both ultra-processed dinner products, snacks \& soft drinks and fast foods among ethnic- compared to non-ethnic Norwegians. As processed foods tend to be less expensive than most fresh foods and ethnic minorities in Western societies often belong to low-income groups with lower living standards than the majority population [45], these findings were rather unexpected. Nevertheless, only $10 \%$ of the study sample was non-ethnic Norwegians and countries of origin for these participants were unknown. It is reasonable to assume that this small group was not representative of all non-ethnic parents in Norway, and furthermore there might be differences in UPFC in non-ethnic Norwegians with different countries of origin.

In the current study, a strong positive association was found between having three or more children in the household and consumption of ultra-processed food products, but not snacks \& soft-drinks and fast foods, compared to having only one child in the household.

In contrast to our study, another study has confirmed that households with one child consumed more fast foods than households with no children and that households with more than one child consumed less fast foods than households with only one child [46].

Finally, adjusted analyses in the present study showed higher odds of consuming ultra-processed dinner products and fast foods, but not snacks \& soft drinks, among overweight/obese participants compared to normal weight participants. A few previously published studies have assessed the potential association between weight status and intake of highly processed foods $[6,7,12,14]$, though the majority of these have investigated food intake as a predictor of overweight/obesity, rather than an outcome.

\section{Strengths and limitations}

An important strength of the current study was the use of a validated measure on time scarcity. The use of three separate scores indicative of UPFC (ultra-processed dinner products, sweet/salty snacks \& soft drinks and fast foods away from home) was also considered to be a strength, as it is reasonable to assume that there might be different factors influencing consumption of e.g. ready-meals and soft drinks. To the best of our knowledge, no previous studies have investigated the effect of time scarcity on UPFC, and the current study might therefore provide valuable input when developing future interventions and nutritional strategies. Nevertheless, there were also some study limitations. The response 
rate was only $16 \%$, and the study sample was overrepresented by highly educated females. Due to the lack of representativeness in the study sample, analyses with appropriate sampling weights (for gender and educational level) were conducted.

Also, data collection was conducted in only two of Norway's nineteen counties, thus findings in this study are not necessarily representative of Norwegian kindergarten parents in general. Regarding the operationalisation of UPFC, it is possible that other food classifications than NOVA could have been more appropriate. Also, the grouping of food items used as indicators of UPFC could have been more precise, especially for the score measuring consumption of fast foods away from home. Furthermore, the questionnaire was pilot-tested in seven subjects from a corresponding population of parents of toddlers, yet it is a limitation that the UPFC scores have not been validated. As we analysed cross-sectional data, conclusions regarding cause and effect cannot be drawn. Additionally, all data was based on self-report questionnaires, which might have increased the risk of social desirability bias. Considering sociodemographic, the variables were not tested independently, but entered as covariates in the logistic model.

\section{Conclusions}

Findings in the present study suggest that time scarcity is associated with an increased consumption of UPF, including ultra-processed dinner products, snacks \& soft drinks and fast foods, after adjusting for sociodemographic correlates and weight status. Furthermore, gender, ethnicity, educational level, number of children in the household and weight status were identified as important factors associated with the consumption of certain types of UPF. Future studies with a longitudinal design are warranted in order to further explore the relationship between time scarcity and UPFC.

\section{Endnote}

${ }^{1} \mathrm{~A}$ name, not an acronym

\section{Additional file}

Additional file 1: HSL Questionnaire. Questionnaire. English translation of the combined questionnaire from the cross-sectional studies The Healthy and Sustainable Lifestyle project and the Child Food Courage project. (ZIP $293 \mathrm{~kb})$

\section{Abbreviations}

BMI: Body Mass Index; Cl: Confidence interval; OR: Odds ratio; SD: Standard deviation; UPF: Ultra-processed foods; UPFC: Ultra-processed food consumption

Acknowledgements

Not applicable.

\section{Funding}

University of Agder and Norwegian Women's Public Health Association financed the present study, but were not involved in designing the study, collection, analyses and interpretation of data or in writing the manuscript.

Availability of data and materials

We do not wish to share our data prior to project completion.

\section{Authors' contributions}

THS, EB, NCØ and MKT conceived the Healthy and Sustainable Lifestyle-project. HBTB, SHH, NCØ and EB designed the cross-sectional study and created the questionnaire. HBTB and SHH conducted the survey. ILD, CBN, EB and THS developed ideas as well as the scores and statistical models presented in this paper. ILD, CBN and THS drafted the manuscript. All authors critically revised the article and approved the final version of the manuscript.

Authors' information

ILD and CBN are joint first authors.

Competing interests

The authors declare that they have no competing interests.

Consent for publication

Not applicable.

Ethics approval and consent to participate

Research clearance was obtained from Norwegian Social Science Data Services. Written informed consent was obtained from participants as they chose to respond to the online survey in the Healthy Sustainable Lifestyle project.

\section{Publisher's Note}

Springer Nature remains neutral with regard to jurisdictional claims in published maps and institutional affiliations.

Received: 3 October 2016 Accepted: 4 May 2017

Published online: 15 May 2017

\section{References}

1. Monteiro CA, et al. A new classification of foods based on the extent and purpose of their processing. Cadernos Saude Publica. 2010;26(11):2039-49.

2. Monteiro $C$, et al. The food system. Food classification. Public health. NOVA. The star shines bright. World Nutr. 2016;7(1-3):28-38.

3. Monteiro CA, et al. Ultra-processed products are becoming dominant in the global food system. Obes Rev. 2013;14(Suppl 2):21-8.

4. Moubarac JC, et al. Consumption of ultra-processed foods and likely impact on human health. Evidence from Canada. Public Health Nutr. 2013;16(12): 2240-8

5. Monteiro CA, et al. Increasing consumption of ultra-processed foods and likely impact on human health: evidence from Brazil. Public Health Nutr. 2011;14(1):5-13.

6. Alkerwi A, Crichton GE, Hebert JR. Consumption of ready-made meals and increased risk of obesity: findings from the observation of cardiovascular risk factors in Luxembourg (ORISCAV-LUX) study. Br J Nutr. 2015;113(2):270-7.

7. Pereira MA, et al. Fast-food habits, weight gain, and insulin resistance (the CARDIA study): 15-year prospective analysis. Lancet. 2005;365(9453):36-42.

8. Tavares LF, et al. Relationship between ultra-processed foods and metabolic syndrome in adolescents from a Brazilian family doctor program. Public Health Nutr. 2012;15(1):82-7.

9. Bouvard V, et al. Carcinogenicity of consumption of red and processed meat. Lancet Oncol. 2015;16(16):1599-600.

10. Chan DS, et al. Red and processed meat and colorectal cancer incidence: meta-analysis of prospective studies. PLoS One. 2011;6(6):e20456.

11. Greenwood DC, et al. Association between sugar-sweetened and artificially sweetened soft drinks and type 2 diabetes: systematic review and doseresponse meta-analysis of prospective studies. Br J Nutr. 2014;112(5):725-34.

12. Malik VS, Schulze MB, Hu FB. Intake of sugar-sweetened beverages and weight gain: a systematic review. Am J Clin Nutr. 2006;84(2):274-88.

13. Malik VS, et al. Sugar-sweetened beverages and risk of metabolic syndrome and type 2 diabetes: a meta-analysis. Diabetes Care. 2010;33(11):2477-83. 
14. Malik VS, et al. Sugar-sweetened beverages and weight gain in children and adults: a systematic review and meta-analysis. Am J Clin Nutr. 2013;98(4):1084-102.

15. Micha R, Michas G, Mozaffarian D. Unprocessed red and processed meats and risk of coronary artery disease and type 2 diabetes-an updated review of the evidence. Curr Atheroscler Rep. 2012;14(6):515-24.

16. Micha R, Wallace SK, Mozaffarian D. Red and processed meat consumption and risk of incident coronary heart disease, stroke, and diabetes mellitus: a systematic review and meta-analysis. Circulation. 2010;121(21):2271-83

17. Popkin BM, Adair LS, Ng SW. Global nutrition transition and the pandemic of obesity in developing countries. Nutr Rev. 2012;70(1):3-21.

18. Slimani $\mathrm{N}$, et al. Contribution of highly industrially processed foods to the nutrient intakes and patterns of middle-aged populations in the European prospective investigation into cancer and nutrition study. Eur J Clin Nutr. 2009;63(Suppl 4):S206-25.

19. Moubarac JC, et al. Processed and ultra-processed food products: consumption trends in Canada from 1938 to 2011. Can J Diet Pract Res. 2014;75(1):15-21

20. Poti JM, et al. Is the degree of food processing and convenience linked with the nutritional quality of foods purchased by US households? Am J Clin Nutr. 2015:101(6):1251-62.

21. Martinez Steele E, et al. Ultra-processed foods and added sugars in the US diet: evidence from a nationally representative cross-sectional study. BMJ Open. 2016;6(3):e009892

22. Jabs J, Devine CM. Time scarcity and food choices: an overview. Appetite. 2006:47(2):196-204.

23. Jabs J, et al. Trying to find the quickest way: employed mothers' constructions of time for food. J Nutr Educ Behav. 2007:39(1):18-25.

24. Slater J, et al. Trying to make it all come together': structuration and employed mothers' experience of family food provisioning in Canada. Health Promot Int. 2012;27(3):405-15.

25. Brunner TA, van der Horst K, Siegrist M. Convenience food products. Drivers for consumption Appetite. 2010;55(3):498-506.

26. van der Horst $\mathrm{K}$, et al. A systematic review of environmental correlates of obesity-related dietary behaviors in youth. Health Educ Res. 2007;22(2):203-26.

27. Ohly $\mathrm{H}$, et al. Parental food involvement predicts parent and child intakes of fruits and vegetables. Appetite. 2013;69:8-14.

28. Deshmukh-Taskar $\mathrm{P}$, et al. Does food group consumption vary by differences in socioeconomic, demographic, and lifestyle factors in young adults? The Bogalusa heart study. J Am Diet Assoc. 2007;107(2):223-34.

29. Eicher-Miller HA, Fulgoni VL, Keast DR. Energy and nutrient intakes from processed foods differ by sex, income status, and race/ethnicity of US adults. J Acad Nutr Diet. 2015;115(6):907-18. e6

30. Næss $\varnothing$, et al. Sosial epidemiologi : sosiale årsaker til sykdom og helsesvikt. Oslo: Gyldendal Akademisk; 2009.

31. Thornton LE, Bentley RJ, Kavanagh AM. Individual and area-level socioeconomic associations with fast food purchasing. J Epidemiol Community Health. 2011; 65(10):873-80.

32. Larson N, Story M. A review of environmental influences on food choices. Ann Behav Med. 2009;38(Suppl 1):S56-73.

33. Paeratakul S, et al. Fast-food consumption among US adults and children: dietary and nutrient intake profile. J Am Diet Assoc. 2003; 103(10):1332-8

34. Moubarac JC, et al. Food classification systems based on food processing: significance and implications for policies and actions: a systematic literature review and assessment. Curr Obes Rep. 2014;3(2):256-72.

35. Van Der Lippe T. Dutch workers and time pressure: household and workplace characteristics. Work Employ Soc. 2007;21(4):693-711.

36. Garhammer M. Pace of life and enjoyment of life. J Happiness Stud. 2002; 3(3):217-56

37. Beshara $M$, Hutchinson A, Wilson C. Preparing meals under time stress. The experience of working mothers. Appetite. 2010;55(3):695-700

38. Welch $\mathrm{N}$, et al. Is the perception of time pressure a barrier to healthy eating and physical activity among women? Public Health Nutr. 2009;12(7):888-95.

39. Darian JC, Chicago J. Segmenting by consumer time shortage. J Consum Mark. 1995:12(1):32.

40. de Boer $M$, et al. The influence of lifestyle characteristics and beliefs about convenience food on the demand for convenience foods in the Irish market. Food Qual Prefer. 2004;15(2):155-65.

41. Verlegh PWJ, Candel MJJM. The consumption of convenience foods: reference groups and eating situations. Food Qual Prefer. 1999;10(6):457-64.
42. Silliman K, Rodas-Fortier K, Neyman M. A survey of dietary and exercise habits and perceived barriers to following a healthy lifestyle in a college population. Calif J Health Promot. 2004;2(2):10-9.

43. Larson $\mathrm{Nl}$, et al. Fast food intake: longitudinal trends during the transition to young adulthood and correlates of intake. J Adolesc Health. 2008;43(1):79-86.

44. Davison J, et al. Correlates of food choice in unemployed young people: the role of demographic factors, self-efficacy, food involvement, food poverty and physical activity. Food Qual Prefer. 2015;46:40-7.

45. Jenum AK. In: Næss $\varnothing$, et al., editors. Etniske og kulturelle faktorers betydning for helse, in Sosial epidemiologi : sosiale årsaker til sykdom og helsesvikt. Oslo: Gyldendal Akademisk; 2009.

46. Akbay C, Tiryaki GY, Gul A. Consumer characteristics influencing fast food consumption in Turkey. Food Control. 2007;18(8):904-13.

\section{Submit your next manuscript to BioMed Central and we will help you at every step:}

- We accept pre-submission inquiries

- Our selector tool helps you to find the most relevant journal

- We provide round the clock customer support

- Convenient online submission

- Thorough peer review

- Inclusion in PubMed and all major indexing services

- Maximum visibility for your research

Submit your manuscript at www.biomedcentral.com/submit 